\title{
Reexpansion pulmonary edema in a young lady post- intercostal drainage tube insertion

\author{
Avinash $\mathrm{RM}^{1,{ }^{1},}$, Ashraf Ali Poovil ${ }^{1}$, Nagendra $\mathrm{D}^{1}$, Yashwant $\mathrm{KO}^{1}$, and Latha Sarma ${ }^{1}$ \\ ${ }^{1}$ Department Pulmonology, Krishna Institute Of Medical Sciences Ltd, Ministers road, Secunderabad -500003, Telangana, India
}

\begin{abstract}
Reexpansion pulmonary edema (REPE) is a rare and serious complication after sudden expansion of a collapsed lung, usually after intercostal drainage (ICD) tube insertion for massive pleural effusion or tension pneumothorax or therapeutic thoracocentesis. Symptoms include severe persistent cough, with frothy sputum and dyspnea.It can occur immediately or within few hours after ICD insertion. Risk factors include duration of collapse more than $72 \mathrm{hrs}$, large volume pleural fluid drainage, application of high negative pressure during drainage. Increased permeability of pulmonary capillaries as seen in ARDS (Acute respiratory distress syndrome) is the proposed pathophysiology. We describe a case of REPE in a young female with large left pleural effusion and its successful management.
\end{abstract}

Keywords: reexpansion pulmonary edema; thoracentesis; pleural effusion

*Corresponding author: Avinash RM, Department of Pulmonology, Krishna Institute Of Medical Sciences Ltd, Ministers road, Secunderabad -500003, Telangana, India. Tel.: +91 9790925624; Email: avinash.rm1993@gmail.com

Received 22 March 2018; Revised 05 June 2018; Accepted 19 June 2018; Published 26 June 2018

Citation: Avinash RM, Poovil AA, Nagendra D, Yashwant KO, and Sarma L. Reexpansion pulmonary edema in a young lady postintercostal drainage tube insertion. J Med Sci Res. 2018; 6(3): 9395. DOI: http://dx.doi.org/10.17727/JMSR.2018/6-16

Copyright: (c) 2018 Avinash RM et al. Published by KIMS Foundation and Research Center. This is an open-access article distributed under the terms of the Creative Commons Attribution License, which permits unrestricted use, distribution, and reproduction in any medium, provided the original author and source are credited.

\section{Case report}

21-year-old female presented with swelling of lateral side of left chest for 2 months, shortness of breath for 5 days. The swelling in the left infrascapular area had no signs of inflammation. She was found to have large Lt pleural effusion on chest X-ray (Figure 1). After informed consent, intercostal drainage tube was inserted on left side. As the intercostal drainage tube (ICD) was blocked, she was asked to cough. Immediately she developed severe cough with mucoid sputum, mild desaturation. Cough persisted for one hour, reducing slowly over next 1-2 hrs. Post-ICD check X-ray showed dense consolidation with peripheral distribution over expanded Lt lung (Figure 2). A chest computed tomography (CT) scan showed dense consolidation with air bronchogram, ground glass opacities and interlobular septal thickening in the left lung suggestive of possible re-expansion pulmonary edema and extensive subcutaneous emphysema along the entire left 
chest wall extending up to the neck with minimal pneumomediastinum (Figure 3). Her symptoms subsided slowly over next few hours. Pleural fluid analysis was s/o exudate with high ADA levels and GeneXpert did not detect MTB. Serial chest $\mathrm{x}$ rays showed significant improvement (Figure 4) and ICD was removed when there was no drainage after 5 days. Fine needle aspiration of left chest lesion detected Mycobacterium tuberculosis by GeneXpert method and she was promptly started on ATT.

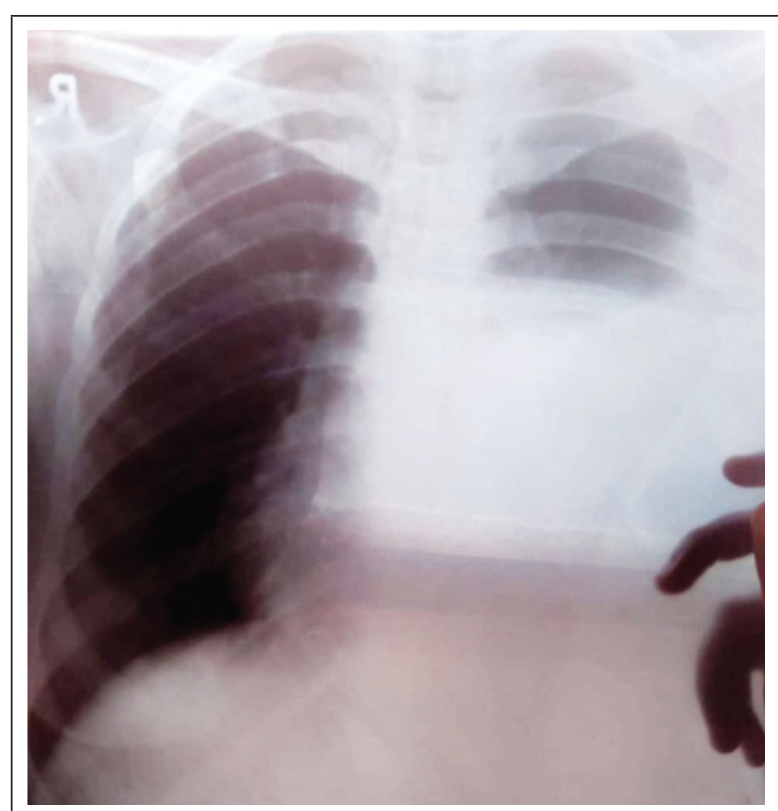

Figure 1: Initial chest X-ray shows left large pleural effusion.

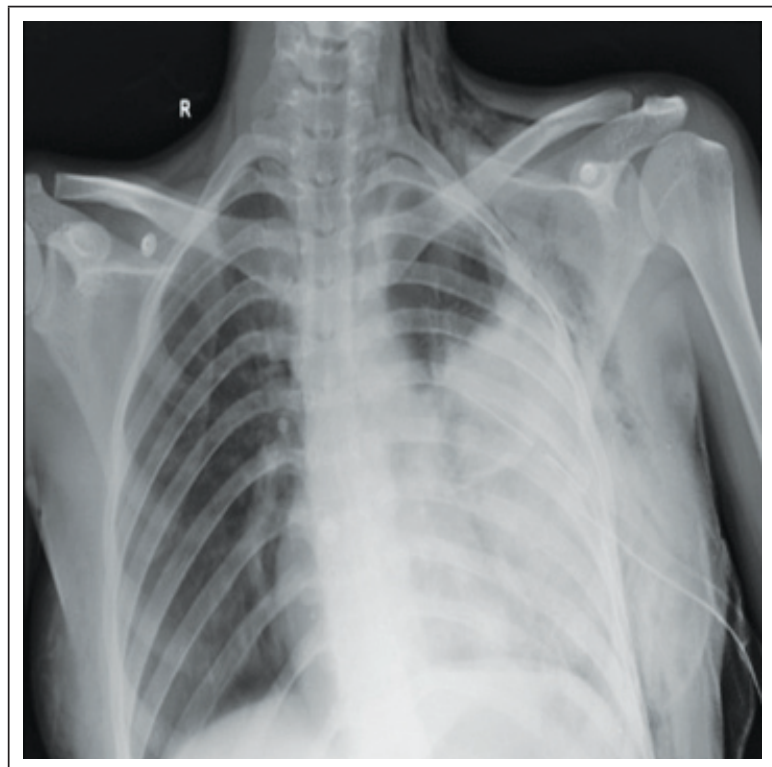

Figure 2: Chest X-ray after ICD insertion shows left midzone \& lower zone consolidation with left ICD in situ.
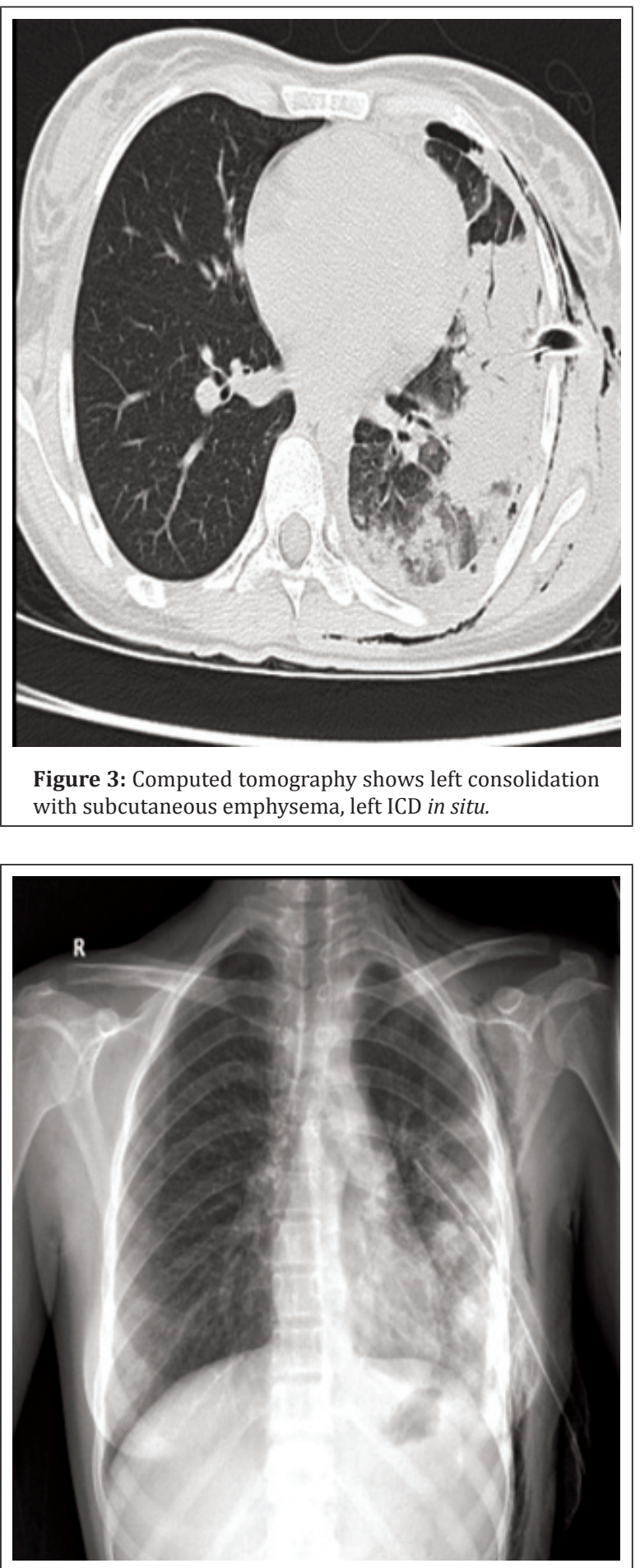

Figure 4: Chest X-ray before removal of ICD tube.

\section{Discussion}

REPE is a rare but life threatening condition, usually occurs unilaterally after reexpansion of a collapsed lung [1]. Incidence ranges between 1 - 14\% [2]. Its pathogenesis is multifactorial [3]. Proposed mechanisms include direct injury from surfactant 
dysfunction in chronic atelectatic lung and release of inflammatory mediators (IL8, MCP1, NO, free radicals) which perpetuate microvascular lesions, also altering capillary permeability [4-6] increased transpleural pressures when excessive negative pleural pressures are created during removal of fluid or air in the setting of an unexpandable lung, or indirect injury from reperfusion. The risk factors include younger age, female sex, greater degree \& longer duration of lung collapse, use of negative pressure during treatment and evacuation more than $2000 \mathrm{ml}$ [7]. Patients typically present soon after inciting event (mins to hrs), or can be delayed for up to $24-48$ hours in some cases, features include acute onset dyspnea, cough, hypoxemia, but may vary from asymptomatic radiologic findings to respiratory failure with circulatory shock. Typical CT findings include ipsilateral GGO's, septal thickening, focal consolidation and areas of atelectasis [8]. Sypmtoms and radiographics abnormalities typically resolve over $24-48$ hours. Treatment is supportive mainly consisting of supplemental oxygen, if necessary mechanical ventilation. Prevention is based on careful pleural emptying. A strategy to avoid REPE is monitoring pleural pressure during emptying. Direct measurement of pleural pressure is performed during thoracocentesis, initial mean pleural pressure is measured just after insertion of thoracocentesis catheter into pleural space. During subsequent fluid removal, the pleural pressure is measured after each aliquot (eg., $100-250 \mathrm{ml}$ ) of pleural fluid, it is recommend to stop fluid removal when the mean pleural pressure decreases to -20 $\mathrm{mmH}_{2}$ 0. Symptoms such as persistent cough, thoracic pain, dyspnea must be considered as an indication of need to halt the drainage, because they may indicate a reduction of intrapleural pressure lower than -20 $\mathrm{cmH}_{2} \mathrm{O}$, even reaching $-50 \mathrm{cmH}_{2} \mathrm{O}$ during therapeutic thoracocentesis (9).

In this case, symptoms appeared immediately after procedure with mild desaturation (after removal of $500 \mathrm{ml}$ of fluid). Her symptoms and radiological features improved significantly over a period of 48 hrs without any active intervention.

\section{Conclusion}

Reexpansion pulmonary edema is a rare but dangerous complication following rapid pleural drainage. Early recognition and prompt management is the key to prevent mortality.

\section{Conflicts of interest}

Authors declare no conflicts of interest.

\section{References}

[1] Sohara Y. Reexpansion pulmonary edema. Ann Thorac Cardiovasc Surg. 2008; 14(4):205-209.

[2] Rozenman J, Yellin A, Simansky DA, Shiner RJ. Re-expansion pulmonary oedema following spontaneous pneumothorax. Respir Med. 1996; 90(4):235-238

[3] Shanahan MX, Monk I, Richards HJ. Unilateral pulmonary oedema following re-expansion of pneumothorax. Anaesth Intensive Care. 1975; 3(1):19-30.

[4] Nakamura M, Fujishima S, Sawafuji M, Ishizaka A, Oguma $\mathrm{T}$, et al. Importance of interleukin-8 in the development of reexpansion lung injury in rabbits. Am J Respir Crit Care Med. 2000; 161(3 Pt 1):1030-1036.

[5] Sakao Y, Kajikawa O, Martin TR, Nakahara Y, Hadden WA 3rd, et al. Association of IL-8 and MCP-1 with the development of reexpansion pulmonary edema in rabbits. Ann Thorac Surg. 2001; 71(6):1825-1832.

[6] Suzuki S, Tanita T, Koike K, Fujimura S. Evidence of acute inflamatory response in reexpansion pulmonary edema. Chest. 1992; 101(1):275-276.

[7] Mahfood S, Hix WR, Aaron BL, Blaes P, Watson DC. Reexpansion pulmonary edema. Ann Thorac Surg. 1988; 45(3):340-345

[8] Gleeson T, Thiessen R, Müller N. Reexpansion pulmonary edema: Computed tomography findings in 22 patients. J Thorac Imaging. 2011; 26(1):36-41.

[9] Light RW, Jenkinson SG, Minh V, George RB. Observations on pleural pressures as fluid is withdrawn during thoracentesis. Am Rev Respir Dis. 1980; 121(5):799-804. 